Cite this: RSC Adv., 2017, 7, 22501

Received 12th February 2017

Accepted 5th April 2017

DOI: $10.1039 / \mathrm{c} 7 \mathrm{ra01770g}$

rsc.li/rsc-advances
Check for updates

\section{Decrease in the double layer capacitance by faradaic current}

\author{
Koichi Jeremiah Aoki, (iD *a Jingyuan Chen, (DD*b Xiangdong Zeng (iD) ${ }^{b}$ \\ and Zhaohao Wang (iD ${ }^{b}$
}

This study describes the reverse of the well-known double layer effects on charge transfer kinetics in the relationship between a cause and an effect. The reversible redox reaction of a ferrocenyl derivative decreased the capacitive values of the double layer impedance up to negative values, corresponding to an inductive component. This observation was disclosed by the subtraction of the real admittance from the imaginary admittance, which can extract the net double layer capacitance from the Warburg impedance. The inductance-like behavior is caused due to two reasons: (i) the double layer capacitance in the polarized potential domain is determined by the low concentrations of the fieldoriented solvent dipoles that are considered as the conventionally employed redox concentrations and (ii) the double layer capacitance is caused by the orientation of the dipoles in the same direction as that of the electric field, whereas the redox reaction generates charge in the direction opposite to the field. The faradaic effect was demonstrated via the ac-impedance data obtained for the ferrocenyl compound in a $\mathrm{KCl}$ solution in the unpolarized potential domain between $1 \mathrm{~Hz}$ and $3 \mathrm{kHz}$ frequency. The negative admittance was proportional to the frequency. The theory of negative capacitance was presented by combining the mirror-image surface charge with the Nernst equation.

\section{Introduction}

Double layer (DL) effects on charge transfer kinetics are a classical subject, which was first reported in 1933 by Frumkin. ${ }^{1}$ These effects deal with the variations of the charge transfer rate with the concentration and/or type of supporting electrolyte as well as with the specifically adsorbed electrolyte. ${ }^{2,3}$ In particular, the effect of the supporting electrolyte is called the Frumkin's DL effect. ${ }^{1,4}$ It includes the correction of the potential at the closest approach of the redox species, which may be varied with the concentration of the supporting electrolyte; well-known examples are cadmium ions, ${ }^{5}$ zinc ions, ${ }^{6}$ and some aromatic compounds. ${ }^{7}$ However, there are some ambiguities in the participation of the specifically adsorbed species, ${ }^{8-12}$ in charge interactions, ${ }^{13,14}$ in modification by finite ionic size,${ }^{15-18}$ in the geometrical effects ${ }^{19-23}$ of the Poisson-Nernst-Planck equation, in simplification of the plane of the closest approach, ${ }^{24}$ in complications by ions ${ }^{25-28}$ and by the orientation of the solvent molecules, ${ }^{29}$ in charge distribution within a redox species,${ }^{30}$ and in coupling of the charged redox species and supporting electrolyte. ${ }^{31,32}$ The Frumkin's correction has recently been reviewed from a theoretical point of view. ${ }^{33}$

${ }^{a}$ Electrochemistry Museum, Fukui, 910-0804 Japan

${ }^{b}$ Department of Applied Physics, University of Fukui, 3-9-1 Bunkyo, Fukui, 910-0017 Japan.E-mail: jchen@u-fukui.ac.jp
The fundamental concept of estimating the DL effects is that the faradaic reactions should occur in the DL structure firmly established with solvents and/or salts independent of the reactions. ${ }^{34-37}$ This is based on the intuition that the concentration of the redox species in the voltammetry is much smaller than the concentration of the solvent molecules and/or supporting electrolyte. However, our experimental results on the frequency-dependence of the DL impedance indicate that (i) the DL capacitance is independent of the concentration of the salt, ${ }^{38,39}$ the type of the salt ${ }^{40}$ and the dcpotential; ${ }^{40,41}$ (ii) it is caused by the field-driven orientation of the solvent molecules on the electrode; ${ }^{42}$ and (iii) the interaction energy of the dipole-dipole of solvent molecules or hydrogen bonding energy is larger by one order in magnitude than the externally driven orientation energy such that a small molar concentration of the solvent molecules participate in the DL impedance. ${ }^{43,44}$ The behavior (i) with regard to the frequency dispersion is in accordance with the results reported in the literature. ${ }^{45-49}$ The frequency-dependence has been attributed to the microscopic surface roughness, ${ }^{50,51}$ non-uniform current distribution on the electrode surfaces, ${ }^{52-55}$ fractal surface geometry, ${ }^{56-59}$ and molecular interaction on the electrode surfaces. ${ }^{43}$ It is necessary to take into account the relationship between the DL impedance and the faradaic impedance without intuition. A technical problem is how to separate the two impedances without 
ambiguity, especially as for the frequency-dependence of the DL capacitance and electrode kinetics.

A simple redox reaction is generally controlled by both the charge transfer step and diffusional step. The former is possibly complicated with the DL impedance because of involvement of a surface process, whereas the latter may be clearly distinguished from the DL effect because the process is carried out in the solution phase. Thus, we first examined the latter, i.e. the effects of the Warburg impedance on the DL impedance. If both impedances are independent of each other, they can be represented as a parallel equivalent circuit, as depicted in Fig. 1(A). A real part of the Warburg impedance for infinite diffusion has the same value as that of the imaginary part, according to the solution of the diffusion equation, ${ }^{\mathbf{6 0 , 6 1}}$ although there are some complications in the nanoscale diffusion domains. ${ }^{62}$ As a result, the values obtained for the observed real admittance subtracted from those of the imaginary admittance should be those of the DL impedance. This was our fundamental technique to examine the relationship between the Warburg impedance and the DL impedance.

To obtain the relationship, it is necessary to both experimentally and theoretically specify the diffusion-controlled conditions. Herein, we used (ferrocenylmethyl) trimethylammonium (FcTMA) as a redox species with negligible effects of the heterogeneous rates. Thus, the problems such as the (a) accuracy of the impedance data, (b) floating capacitance, (c) participation of harmonic components, (d) participation of the electrode kinetics of FcTMA, and (e) effects of the frequencydependence of the DL capacitance on the Warburg impedance need to be solved in detail. We have discussed (a) and (b) in the Experimental section, (c) in the Appendix, and (d) and (e) in the Results and discussion section.

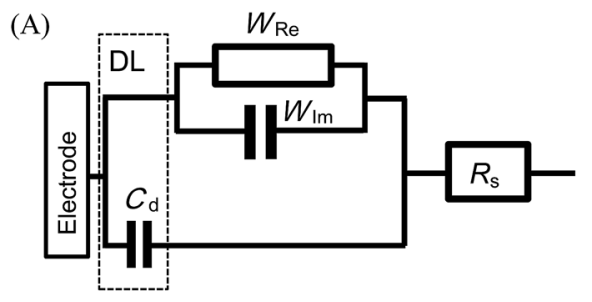

(B)

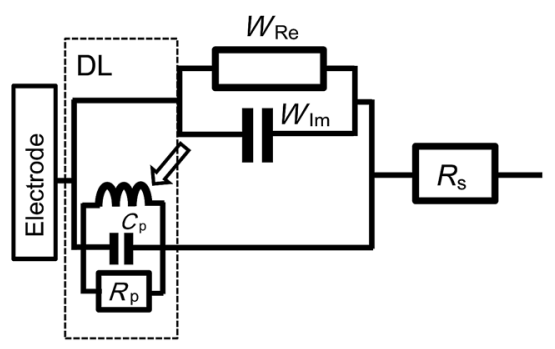

Fig. 1 Equivalent circuits of (A) the conventional combination of the Warburg impedance (real part $W_{\mathrm{Re}}$ and imaginary part $W_{\mathrm{im}}$ ), the double layer capacitance $C_{\mathrm{d}}$ and the solution resistance $R_{\mathrm{S}}$, and (B) the suggested combination including the inductance-like capacitance caused by the faradaic reaction and the parallel $\mathrm{DL}$ impedance with the real $R_{\mathrm{p}}$ and imaginary $C_{p}$ components.

\section{Experimental}

FcTMA purchased from Tokyo Chemical Industry was in the iodide form. Iodide ion was substituted into $\mathrm{PF}_{6}{ }^{-}$by adding $\mathrm{NH}_{4} \mathrm{PF}_{6}$ to $\mathrm{FcTMA}^{+}-\mathrm{I}^{-}$and recrystallizing the product. All the chemicals were of analytical grade. Water was distilled and ionexchanged prior to use.

Since a disk-exposed working electrode often yields large background currents, which depend on the mechanical polish, chemical treatments and pre-electrolysis, the instability was empirically ascribed to the crevices at the boundary between the electrode and the insulator. To avoid the instability, we used a platinum wire electrode, $0.5 \mathrm{~mm}$ in diameter, without shielding just by inserting it into a solution up to a given length ( $c a$. $7.5 \mathrm{~mm}) .{ }^{38-40}$ The length was determined each time when the wire was set in the solution. The surface was polished with buff including alumina powder, subjected to ultrasonication in a solution of mixed acid composed of $\mathrm{H}_{3} \mathrm{PO}_{4}+\mathrm{HNO}_{3}+\mathrm{CH}_{3}$ $\mathrm{COOH}$ (Vol. $2: 1: 1$ ), and then rinsed with distilled water. The length was controlled using an optical Z-stage and was evaluated using an optical microscope. The reference electrode and the counter electrodes were $\mathrm{Ag} \mid \mathrm{AgCl}$ in a saturated $\mathrm{KCl}$ solution and a platinum coil, respectively. The potentiostat used for the acimpedance measurements was a Compactstat (Ivium, the Netherlands). The ac-impedance data slightly varied with the selected current ranges as well as the frequencies of the filter and were confirmed to be consistent within the experimental error.

The concentration of $\mathrm{KCl}$ was $1 \mathrm{M}\left(=\mathrm{mol} \mathrm{dm}{ }^{-3}\right)$. The solution resistance was determined from the intercept of the real impedance at zero imaginary resistance in the Nyquist plot. The solution resistance at the electrode, $7.6 \mathrm{~mm}$ long, was $(3.2 \pm 0.2)$ $\Omega$ for a series of ac-experimental runs carried out by changing the dc-voltage.

The delay of the potentiostat was examined using a series combination of a carbon resistance $(1,10 \mathrm{k} \Omega$ ) and a film capacitor $(0.1 \mu \mathrm{F})$ over the frequency range from $1 \mathrm{~Hz}$ to $10 \mathrm{kHz}$. No abnormality was observed so far as $\left|Z_{2}\right| / Z_{1}>0.04$. For these high frequencies that yield $\left|Z_{2}\right| / Z_{1}<0.04$, the $\left|Z_{2}\right|$ values were overestimated by a few percentages. Most of the experimental conditions of the DL impedance were in the domain of $\left|Z_{2}\right| / Z_{1}>$ 0.04 . When the frequency was over $5 \mathrm{kHz}$, the real part of the impedance was observed to be larger than the calculated values, which was attributed to the delay of the potentiostat decreasing the current. We analyzed the impedance data in the frequency domain from $1 \mathrm{~Hz}$ to $3 \mathrm{kHz}$. This domain will be discussed to present the faradaic effect.

\section{Results and discussion}

\subsection{The presence of negative capacitance in the DL}

The ac-impedance was obtained for several concentrations of FcTMA at various dc-potentials in the ac frequency $f$ ranging from $1 \mathrm{~Hz}$ to $3 \mathrm{kHz}$ at the Pt wire electrode. Fig. 2 shows the Nyquist plots (the imaginary impedance, $Z_{2}$, vs. the real one, $Z_{1}$ ) for three dc-potentials. The plots (a) at which the FcTMA was electroinactive were almost overlapped with those without FcTMA in a $\mathrm{KCl}$ solution. They overlapped the lines for which 


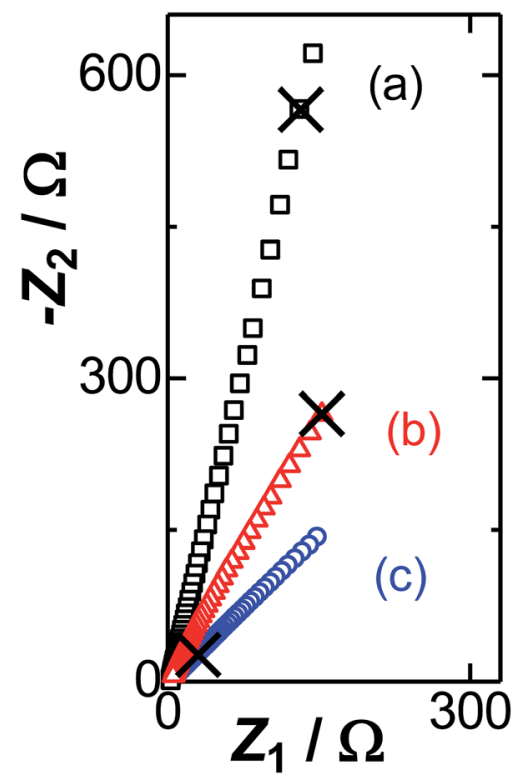

Fig. 2 The Nyquist plots obtained in $3 \mathrm{mM} \mathrm{FcTMA}+1 \mathrm{M} \mathrm{KCl}$ solution at $E_{\mathrm{dc}}=$ (a) 0.1 , (b) 0.3 , and (c) $0.4 \mathrm{~V} \mathrm{vs}$. Ag|AgCl. ( $\times$ ) denote the values at $f$ $=30 \mathrm{~Hz}$

the values of the slope were over 5 . Finite values of the slopes, as shown in Fig. 2, instead of a vertical line suggests the CPE behavior $^{63-66}$ or the power law of the frequency-dependence. ${ }^{38-42}$ When the dc potential was in the electroactive domain (b) of FcTMA, the values of $-Z_{2}$ were smaller than those in the inactive potential domain (a), due to the contribution of the faradaic current. The plot at the formal potential (c) exhibited a line for which the slope value was one. The equi-values of $Z_{1}$ and $-Z_{2}$ suggest the Warburg impedance. No hemi-circle due to the kinetic control was observed even at a frequency as high as 5 $\mathrm{kHz}$. Consequently, the impedance for (c) should be controlled by the diffusion current of FcTMA. The diffusion-controlled step was confirmed using cyclic voltammetry by the proportionality of the peak currents to the square-roots of the scan rates less than $0.5 \mathrm{~V} \mathrm{~s}^{-1}$ in $0.4-3.0 \mathrm{mM}$ FcTMA solutions. The interaction between the DL impedance and the Warburg impedance may be revealed in the variation of their admittance with the frequency rather than the Nyquist plots because the two impedances can be regarded as a parallel combination in the equivalent circuit (Fig. 1(A)).

We considered the equivalent circuit at which $C_{\mathrm{d}}$ exhibited frequency-dependence when the ac voltage $V_{\text {ac }}=V_{0} \mathrm{e}^{\mathrm{i} \omega t}$ was applied to the electrode, where $V_{0}$ is the amplitude of the acvoltage, $\omega$ is the angular velocity of the ac-voltage, and $i$ is the imaginary unit. The current density through the frequencydependent DL impedance can be represented in terms of the time-derivative of the charge density $\sigma_{\mathrm{D}}\left(=C_{\mathrm{d}} V_{\mathrm{ac}}\right)$ stored in the DL capacitance as ${ }^{38}$

$$
j_{\mathrm{DL}}=\mathrm{d} \sigma_{\mathrm{D}} / \mathrm{d} t=C_{\mathrm{d}} \mathrm{d} V_{\mathrm{ac}} / \mathrm{d} t+V_{\mathrm{ac}} \mathrm{d} C_{\mathrm{d}} / \mathrm{d} t
$$

The first term becomes $\mathrm{i} \omega C_{\mathrm{d}} V_{\mathrm{ac}}$, whereas the second term can be rewritten as $V_{\mathrm{ac}}\left(\mathrm{d} C_{\mathrm{d}} / \mathrm{d} \omega\right)\left(\mathrm{d} \omega / \mathrm{d} t^{\prime}\right)$, where $\omega=1 / t^{\prime}$. When the empirical expression for the frequency-dependence $f^{-\lambda}$ at the frequency $f(=\omega / 2 \pi)$ and a positive constant of $\lambda$ (ref. 38, 39 and 41) are inserted into eqn (1), the DL admittance is given by

$$
Y_{\mathrm{DL}}=(\lambda+\mathrm{i}) \omega C_{\mathrm{p}}
$$

where $C_{\mathrm{d}}$ is mentioned as $C_{\mathrm{p}}$ to stress the parallel combination of the real admittance $\left(\lambda \omega C_{\mathrm{p}}\right)$ and the imaginary admittance $\left(\omega C_{\mathrm{p}}\right)$ in the equivalent circuit.

On the other hand, the faradaic ac-current density is given by $^{61}$

$$
j_{\mathrm{F}}=(1+\mathrm{i}) c^{*} F^{2} \sqrt{D \omega} V_{0} \mathrm{e}^{\mathrm{i} \omega t}\left[\sqrt{2} R T \cosh ^{2}\left(\zeta_{\mathrm{dc}} / 2\right)\right]^{-1}
$$

with

$$
\zeta_{\mathrm{dc}}=\left(E-E^{0}\right) F / R T
$$

where $c^{*}$ is the sum of the bulk concentration of the oxidized species and the reduced species and $D$ is the diffusion coefficient common to both species. Eqn (3) is valid on the assumption of neglecting harmonics. It is necessary to examine the assumption for eqn (3) before analyzing the experimental data. The derivation of eqn (3) using the Laplace transformation has been described in the Appendix. The derivation shows that eqn (3) is valid within $8 \mathrm{mV}$ of the $E_{\mathrm{dc}}$ error at $V_{0}=10 \mathrm{mV}$ even if the current includes harmonic components. If the DL impedance is considered to be independent of the Warburg impedance, the observed current is a simple sum of the currents through the DL and through the Warburg impedance, as illustrated in Fig. 1(A). Then, the real admittance $Y_{1}$ and the imaginary admittance $Y_{2}$ are given, respectively, by

$$
\begin{gathered}
Y_{1}=\lambda \omega C_{\mathrm{p}}+Y_{\mathrm{W}} \\
Y_{2}=\omega C_{\mathrm{p}}+Y_{\mathrm{W}}
\end{gathered}
$$

where

$$
Y_{\mathrm{W}}=c^{*} F^{2} \sqrt{D \omega}\left[\sqrt{2} R T \cosh ^{2}\left(\zeta_{\mathrm{dc}} / 2\right)\right]^{-1}
$$

We determined the numerical values of $Y_{1}$ and $Y_{2}$ using the following equation:

$$
Y_{1}=\left(Z_{1}-R_{\mathrm{S}}\right) /\left[\left(Z_{1}-R_{\mathrm{S}}\right)^{2}+Z_{2}^{2}\right], Y_{2}=Z_{2} /\left[\left(Z_{1}-R_{\mathrm{S}}\right)^{2}+Z_{2}^{2}\right]
$$

where the solution resistance $R_{\mathrm{S}}$ was determined from the intercept of $Z_{1}$ in the Nyquist plot by extrapolation to $-Z_{2} \rightarrow 0$. According to eqn (4) and (5), the subtraction $Y_{2}-Y_{1}$ cancels the Warburg impedance. When the empirical frequencydependence

$$
C_{\mathrm{p}}=C_{\mathrm{p}, 1 \mathrm{~Hz}} f^{-\lambda}
$$

is inserted into the subtraction, we obtain

$$
Y_{2}-Y_{1}=(1-\lambda) \omega C_{\mathrm{p}}=2 \pi(1-\lambda) C_{\mathrm{p}, 1 \mathrm{~Hz}} f^{1-\lambda}
$$




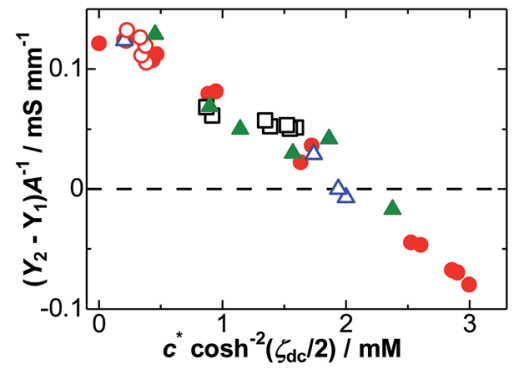

Fig. 3 The dependence of $\left(Y_{2}-Y_{1}\right) / A$ at $f=100 \mathrm{~Hz}$ on the surface concentration causing ac currents in the solution including (filled circles) $3 \mathrm{mM}$, (filled triangles) $2.4 \mathrm{mM}$, (triangles) $2 \mathrm{mM}$, (squares) $1.6 \mathrm{mM}$, and (circles) $0.4 \mathrm{mM}$ FcTMA, where $E^{0}=0.401 \mathrm{~V} v \mathrm{vs} . \mathrm{Ag} \mid \mathrm{AgCl}$ and $A$ is the area of the electrode.

Fig. 3 shows the variations of $Y_{2}-Y_{1}$ at $f=100 \mathrm{~Hz}$ with the dc-surface concentration $c^{*} \cosh ^{-2}\left(\zeta_{\mathrm{dc}} / 2\right)$. The values of $Y_{2}-Y_{1}$ decreased with an increase in the dc-surface concentration. Those at concentrations larger than $2 \mathrm{mM}$ were decreased to negative values. The variation, as shown in Fig. 3, is the intuitive proof of the dependence of the DL capacitance on the faradaic reaction.

Fig. 3 predicts that the values of $Y_{2}-Y_{1}$ depend on the frequency. Fig. 4 shows the logarithmic variations with the frequency at two dc-potentials. The plots at the formal potential of FcTMA (0.4 V, (b)) show a slope of 0.5 for the Warburg impedance, in accordance with eqn (6). In contrast, the plots for the partial participation (a) of the faradaic reaction show a change of 0.5 in the slopes at a low frequency and 1 at a high frequency. The former is controlled by the Warburg impedance, whereas the latter is controlled by the DL impedance. The plots of $\log Y_{2}$ against $\log f$ are approximately linear for all the dcpotentials.

Fig. 5 shows variations of $\left|Y_{2}-Y_{1}\right|$ with $f$ on a logarithmic scale for $c^{*}=3 \mathrm{mM}$. The values of $Y_{2}-Y_{1}$ for $0.38 \mathrm{~V}<E_{\mathrm{dc}}<$ $0.42 \mathrm{~V}$ were negative over the frequency domain. This was not due to errors in the subtraction because $0.06<\left|Y_{2}-Y_{1}\right| / Y_{2}<0.2$. The negative values indicate that $(1-\lambda) C_{\mathrm{p}}<0$ (from eqn (8)). If 1 $-\lambda<0$ and $C_{\mathrm{p}}>0$, the admittance given by $\omega C_{\mathrm{p}}=2 \pi C_{\mathrm{p}, 1 \mathrm{~Hz}} f^{1-\lambda}$

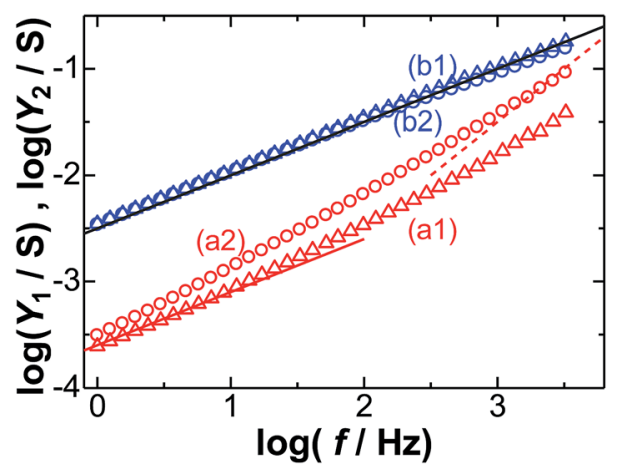

Fig. 4 The variations of $(a 1, b 1) \log \left(Y_{1}\right)$ and $(a 2, b 2) \log \left(Y_{2}\right)$ with $\log f$ at $E_{\mathrm{dc}}=(\mathrm{a}) 0.30 \mathrm{~V}$ and (b) $0.40 \mathrm{~V}$ vs. AglAgCl for $\mathrm{c}^{*}=3 \mathrm{mM}$. The slopes of the solid line and the dashed line are 0.5 and 1.0, respectively.

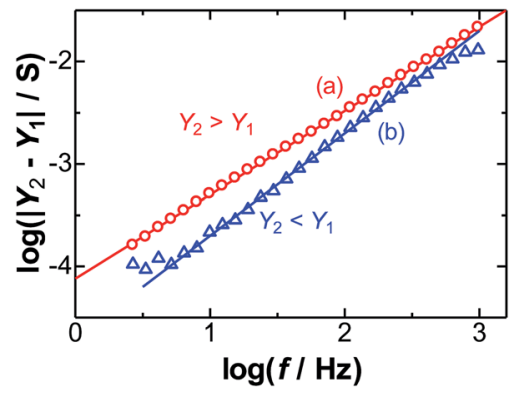

Fig. 5 The variations of $\log \left|Y_{2}-Y_{1}\right|$ with $\log f$ at $E_{\mathrm{dc}}=$ (a) $0.30 \mathrm{~V}$ and (b) $0.40 \mathrm{~V} v \mathrm{v}$. $\mathrm{Ag} \mid \mathrm{AgCl}$ in a solution at $c^{*}=3 \mathrm{mM}$. The slopes of the lines are (a) 0.82 and (b) 1.0 .

might work as an inductance since an increase in the frequency decreases the admittance. If $1-\lambda>0$ and $C_{\mathrm{p}}<0$, the capacitance is an inductance with a negative sign. The negative values have been discussed in the next section. In the dc-potential domain for $Y_{2}-Y_{1}>0$, we evaluated $\lambda$ and $C_{\mathrm{p}, 1 \mathrm{~Hz}}$ from the slope and the intercept, as shown in Fig. 5.

The variations of $\lambda$ and $C_{\mathrm{p}, 1 \mathrm{~Hz}}$ with the dc-potential are shown in Fig. 6, together with the cyclic voltammograms. The values of $\lambda$ are almost independent of the dc-potentials in the potential domains $E_{\mathrm{dc}}<0.36$ and $E_{\mathrm{dc}}>0.44 \mathrm{~V}$, at which the FcTMA is electrochemically inactive. The independence is in accordance with eqn (8). The values of $\lambda$ cannot be determined in the electroactive potential domain because $Y_{2}-Y_{1}<0$. This is a clear demonstration of the presence of the interaction between the DL impedance and the Warburg impedance. A similar variation was found in the dependence of $C_{\mathrm{p}, 1 \mathrm{~Hz}}$ on the dc-potential. The values of $C_{\mathrm{p}, 1 \mathrm{~Hz}}$ suddenly decreased at the boundary between the active and inactive potential domains and become negative in the electroactive potential domain. A negative capacitance is not a remarkable phenomenon. Despite being contentious, this phenomenon has been observed in the ferroelectric superlattices of the films. ${ }^{67-69}$

The observed interaction between two types of the impedance may be due to (i) the low density of dipole moments causing the DL capacitance and (ii) the generation of surface charge via faradaic reactions.

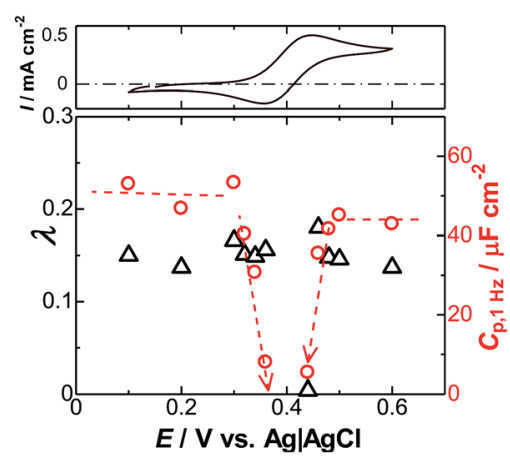

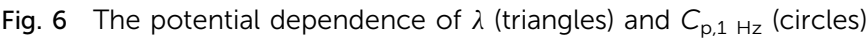
from the depolarized potential domain at $c^{*}=3 \mathrm{mM}$. The upper graph is the cyclic voltammogram of FcTMA at the scan rate $50 \mathrm{mV} \mathrm{s}^{-1}$. 
(i) The orientation of water dipoles by the external electric field, which is mainly responsible for the DL capacitance, is largely suppressed by hydrogen bonding as well as the image force of the dipole on the electrode. ${ }^{43}$ Since the field-orientation energy is one order in magnitude smaller than the hydrogen bonding energy and the imaging force energy, the concentration of the oriented molecules is only $10^{-4}$ times that of the bulk molecules. ${ }^{43,44}$ This corresponds to milli-molar concentration, which is close to the concentration of the redox species in conventional voltammetry. Therefore, the DL capacitance is sensitive to other disturbances such as faradaic currents.

(ii) We temporarily considered oxidation for the faradaic reaction, which generates a charge more positive than that generated by reduction on the electrode. The dipole of the solvent induced by the field is oriented such that it may relax the external field, as illustrated in Fig. 7. In contrast, the charge generated by oxidation enhances the field. This behavior is opposite to the orientation of the dipole and hence the capacitance decreases.

\subsection{The theory of negative capacitance}

First, we define capacitance when the charge density $\sigma_{\mathrm{E}}$ is mounted on one of two parallel electrodes with the distance $L$. The electric field in vacuum is given by $\varphi_{\mathrm{v}}=\sigma_{\mathrm{E}} / \varepsilon_{0}$ using the Gauss law, which provides the voltage $V_{\mathrm{v}}=L \varphi_{\mathrm{v}}$. Then, the capacitance in vacuum is represented by $C_{\mathrm{v}}=\sigma_{\mathrm{E}} / V_{\mathrm{v}}=\varepsilon_{0} / L$. When a solvent with a dipole is inserted into the electrodes, the dipoles are oriented by the field to yield the polarization vector $P_{\mathrm{s}}$, as illustrated in Fig. 7. The polarization uniformly decreases the field by $P_{\mathrm{s}} / \varepsilon_{0}$ to yield the voltage $V_{\mathrm{s}}=L\left(\sigma_{\mathrm{E}}-P_{\mathrm{s}}\right) / \varepsilon_{0}$. Then, the capacitance is given by the following equation:

$$
C_{\mathrm{s}}=\sigma_{\mathrm{E}} / V_{\mathrm{s}}=\varepsilon_{0} / L\left(1-P_{\mathrm{s}} / \sigma_{\mathrm{E}}\right)=\varepsilon_{0} \varepsilon_{\mathrm{d}} / L
$$

where $\varepsilon_{\mathrm{d}}$ is the saturated dielectric constant given by $1 /\left(1-P_{\mathrm{S}} /\right.$ $\left.\sigma_{\mathrm{E}}\right)$. The charge density has almost been demonstrated to be independent of the dc-voltage in the polarized potential domain ${ }^{\mathbf{4 0}}$ because the measured DL capacitance is controlled by the field-oriented dipoles in the Helmholtz layer rather than ion distribution..$^{38,39,42}$

When the elementary charge $e$ is generated near the electrode surface by the electrode reaction, the field profile on the electrode is deformed from lines towards the solution radiating

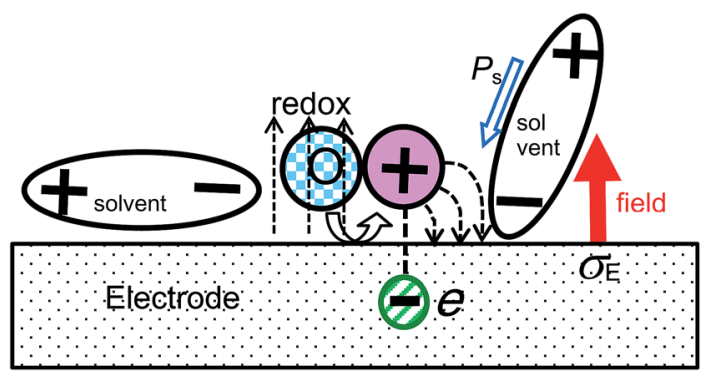

Fig. 7 Schematic of the generation of a positive charge by oxidation, which enhances the field against the orientation of the solvent dipoles. curves around the cation (Fig. 7). The profile can be evaluated using the mirror image technique, ${ }^{70}$ for which a negative charge $-e$ may be located in the electrode symmetrically with respect to the electrode surface. Let the average distance between two neighboring redox species responding to the ac voltage be $l$. Then, the charge density on the electrode becomes $\sigma_{\mathrm{E}}-e / l^{2}$. Since the deformation of the voltage profile is included in the abovementioned charge density, the voltage evaluating the capacitance is $V_{\mathrm{s}}$. Then, the capacitance is expressed by the following equation:

$$
C_{\mathrm{rx}}=\frac{\sigma_{\mathrm{E}}-e / l^{2}}{V_{\mathrm{s}}}=\frac{\sigma_{\mathrm{E}}-e / l^{2}}{L\left(\sigma_{\mathrm{E}}-P_{\mathrm{s}}\right) / \varepsilon_{0}}=\frac{\varepsilon_{0} \varepsilon_{\mathrm{d}}}{L}\left(1-\frac{e}{\sigma_{\mathrm{E}} l^{2}}\right)
$$

If $c^{*}$ is concentration of the molecules, the number of the molecules in a unit volume is $l^{-3}$. When the numbers of the oxidized and reduced species are denoted by $l_{\mathrm{O}}{ }^{-3}$ and $l_{\mathrm{R}}{ }^{-3}$, respectively, the sum is given by $l_{\mathrm{O}}{ }^{-3}+l_{\mathrm{R}}{ }^{-3}=2 c^{*} N_{\mathrm{A}}$, where $N_{\mathrm{A}}$ is the Avogadro constant. The Nernst equation for the ac-voltage is given by $l_{\mathrm{O}}{ }^{-3} / l_{\mathrm{R}}{ }^{-3}=\exp \left(\zeta_{\mathrm{dc}}+\zeta_{\mathrm{ac}}\right)$. Eliminating $l_{\mathrm{R}}{ }^{-3}$ and expressing $l_{\mathrm{O}}{ }^{-3}$ as the Taylor expansion on the assumption of $\left|\zeta_{\text {ac }}\right| \ll\left|\zeta_{\text {dc }}\right|$, we obtain the average ac-voltage $2^{-1 / 2}\left|\zeta_{\text {ac }}\right|$.

$$
l_{\mathrm{O}}{ }^{-3}=2 c^{*} N_{\mathrm{A}}\left(\frac{1}{1+\exp \left(-\zeta_{\mathrm{dc}}\right)}+\frac{2^{-1 / 2}\left|\zeta_{\mathrm{ac}}\right|}{4 \cosh ^{2}\left(\zeta_{\mathrm{dc}} / 2\right)}\right)
$$

Since $l$ is the distance of redox species responding only to the ac-voltage, it should correspond to the second term in eqn (11), which is equal to $l_{\mathrm{R}}{ }^{-3}$. Inserting the second term into eqn (10) yields the following expression:

$$
C_{\mathrm{rx}}=\frac{\varepsilon_{0} \varepsilon_{\mathrm{d}}}{L}\left(1-\frac{e\left(c^{*} N_{\mathrm{A}}\left|\zeta_{\mathrm{ac}}\right|\right)^{2 / 3}}{2 \sigma_{\mathrm{E}} \cosh ^{4 / 3}\left(\zeta_{\mathrm{dc}} / 2\right)}\right)
$$

At high concentrations and at electroactive potentials $\left(\zeta_{\mathrm{dc}} \approx\right.$ 0 ), the second term in the parenthesis is predominant, and hence, the capacitance takes negative values. The negative sign in $C_{\mathrm{rx}}$ means a function of inductance. Zero of the charge density at the potential of zero charge in eqn (12) may make $C_{\mathrm{rx}}$ be minus infinity. We did not find any large negative values for the capacitance in our dc-potential domain. Moreover, we do not have data on the potential of zero charge under our experimental conditions at present.

Eqn (2) shows that the DL admittance has been observed as a sum of the real and the imaginary parts of the current due to the frequency-dependence. Since the diffusion of the redox species is independent of the DL properties, the Warburg impedance can be set in a parallel combination with the DL. Fig. 1(B) shows the predicted equivalent circuit, for which admittance is a simple sum of the five admittances, given by the following equation:

$$
Y / A=(\lambda+\mathrm{i}) \omega C_{\mathrm{p}}+\mathrm{i} \omega C_{\mathrm{rx}}+(1+\mathrm{i}) Y_{\mathrm{W}}
$$

The difference between the real component and the imaginary component is 


$$
-\left(Y_{2}-Y_{1}\right)=2 \pi f X_{\mathrm{A}}
$$

where

$$
X=-C_{\mathrm{rx}}-(1-\lambda) C_{\mathrm{p}} \approx \frac{\varepsilon_{0} \varepsilon_{\mathrm{d}}}{L} \frac{e\left(c^{*} N_{\mathrm{A}}\left|\zeta_{\mathrm{ac}}\right|\right)^{2 / 3}}{2 \sigma_{\mathrm{E}} \cosh ^{4 / 3}\left(\zeta_{\mathrm{dc}} / 2\right)}
$$

on the assumption of high concentrations and $\zeta_{\mathrm{dc}} \approx 0$. Eqn (14) indicates that $Y_{2}-Y_{1}$ should be proportional to $f$ if $X$ is enough large such that the frequency-dependence of $C_{\mathrm{p}}\left(=C_{\mathrm{p}, 1 \mathrm{~Hz}} f^{-\lambda}\right)$ is negligible. The plot in Fig. 5(b) demonstrates the proportionality of $Y_{2}-Y_{1}$ with $f$. The average value of the power of $f$ at different dc-potentials is $1.04 \pm 0.06$ for $0.38 \leq E_{\mathrm{dc}} \leq 0.42 \mathrm{~V} v s$. $\mathrm{Ag} \mid \mathrm{AgCl}$ at $c^{*}=3 \mathrm{mM}$. The unity value of the power means that $C_{\mathrm{rx}}$ is independent of the frequency. When $X$ is not enough large that the frequency-dependence appears as for $c^{*}<2 \mathrm{mM}$, the low and high frequencies may make $-\left(Y_{2}-Y_{1}\right)$ negative and positive, respectively. Then, the plot $\log \left|Y_{2}-Y_{1}\right|$ against $\log f$ should display divergent behavior at the frequency for which $Y_{2}$ $=Y_{1}$, as shown in Fig. 8 where the sign of $Y_{2}-Y_{1}$ changes at $\log f=1.8$. This type of variation is an evident demonstration for the negative capacitance that can be obtained using a single experimental run of varying frequency.

The intercept of the line, as shown in Fig. 5(b), should be $\log (2 \pi X A)$, according to eqn (14). The values of $X$ were plotted against $\cosh ^{-4 / 3}\left(\zeta_{\mathrm{dc}} / 2\right)$ in Fig. 9, falling on one line. The linearity indicates the justification of the present concept of the contribution of the redox charge to the DL capacitance. The slope of the line is $22 \mu \mathrm{F}$, which corresponds to $\left(c^{*} N_{\mathrm{A}}\left|\zeta_{\mathrm{ac}}\right|\right)^{2 / 3} e \varepsilon_{0} \varepsilon_{\mathrm{r}} / 2 L \sigma_{\mathrm{E}}$. For the values of $\varepsilon_{\mathrm{r}}=5$ (ref. 42) and $L=0.1 \mathrm{~nm}$ without surface roughness, we obtained $\sigma_{\mathrm{E}}=3 \times 10^{-4} \mathrm{C} \mathrm{m}^{-2}$, equivalent to the area $\left(22 \mathrm{~nm}^{2}\right)$ occupied by one charge on the electrode. Consequently, the redox charge has a large enough contribution to the DL impedance. If the adsorbed redox charge causes a charge transfer reaction keeping the adsorbed state, only the imaginary admittance should increase. At this point, our result was different from that of the adsorbed charge reported by Unwing's group. ${ }^{71}$

A question arises that why the negative capacitance represented by $Y_{2}<Y_{1}$ was only observed in the high frequency domain, as shown in Fig. 8. This behavior results in the formation of the redox charge density $e / l^{2}$, as shown in eqn (10). Since the formation rate increases in proportion to $f^{1 / 2}$, a higher frequency provides a larger decrease in the capacitance, which

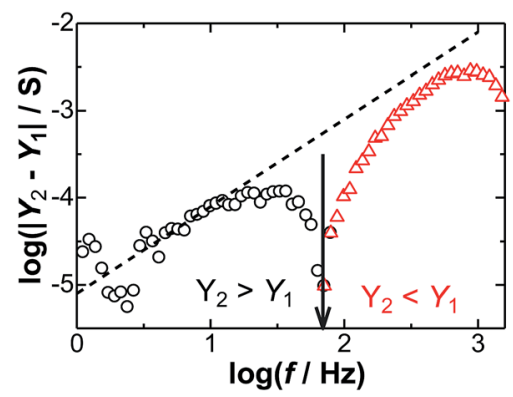

Fig. 8 The variation of $\log \left|Y_{2}-Y_{1}\right|$ with $\log f$ at $E_{\mathrm{dc}}=0.40 \mathrm{~V}$ in a solution at $c^{*}=2 \mathrm{mM}$. The slope of the dashed line is 1 .

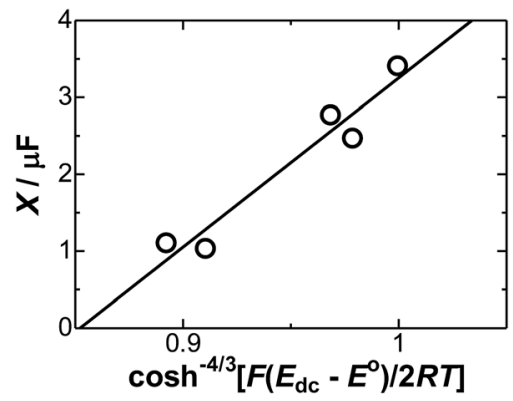

Fig. 9 The variation of $X$ with $\cosh ^{-4 / 3}\left(\zeta_{\mathrm{dc}} / 2\right)$ in a solution of $3 \mathrm{mM}$ FCTMA + $1 \mathrm{M} \mathrm{KCl}$.

mainly contributes to $Y_{2}$. As a result, $Y_{2}<Y_{1}$ is remarkable only in the high frequency region. This observation may also be applied to currents after a short time via chronoamperometry and cyclic voltammetry. The currents may be smaller than the sum of the theoretically evaluated faradaic current and the capacitive current. This will be explored in our future work. The steady-state currents should have no contribution to the DL impedance and hence agree with the theoretically diffusioncontrolled values.

\section{Conclusions}

The DL capacitance can be separated from the Warburg impedance by taking the difference between the real admittance and the imaginary impedance. The extracted DL capacitance decreases with the faradaic current of FcTMA. The decrease is caused by the generation of surface charge via diffusioncontrolled redox reactions, which cancels the DL capacitive charge. This result is opposite to Frumkin's effect in the relationship between an effect and a cause. The parameter for the frequency-dependent power law $\lambda$ was hardly influenced by the redox reactions. When the redox concentration is over $2 \mathrm{mM}$, the DL impedance was observed to be negative. The negative values suggest an inductance, but its frequency-dependence is opposite to that of an ideal coil.

\section{Appendix}

The equation for the ac-impedance controlled by the diffusion of redox species for $\mathrm{R} \leftrightarrow \mathrm{O}+\mathrm{e}^{-}$has been derived herein without taking an infinite limit of the heterogeneous rate constant. The diffusion equations for species $\mathrm{i}=\mathrm{R}$ and $\mathrm{O}$ with a common diffusion coefficient are

$$
\partial c_{\mathrm{i}} / \partial t=D \partial^{2} c_{\mathrm{i}} / \partial x^{2}
$$

The boundary conditions at the electrode surface $(x=0)$ are given by the Nernst equation

$$
\begin{aligned}
\left(c_{\mathrm{O}} / c_{\mathrm{R}}\right)_{x=0} & =\exp \left(\left(E_{\mathrm{dc}}+V_{0} \mathrm{e}^{\mathrm{i} \omega t}-E^{0}\right) F / R T\right) \\
& =\left(c_{\mathrm{O}}^{*} / c_{\mathrm{R}}^{*}\right) \exp \left(\zeta_{\mathrm{ac}}(t)\right)
\end{aligned}
$$

and the balance of the fluxes 


$$
D\left(\partial c_{\mathrm{R}} / \partial x\right)_{x=0}+D\left(\partial c_{\mathrm{O}} / \partial x\right)_{x=0}=0
$$

where $c_{\mathrm{O}}^{*}$ and $c_{\mathrm{R}}^{*}$ are the bulk concentrations and $\zeta_{\mathrm{ac}}(t)=$ $V_{0} \mathrm{e}^{\mathrm{i} \omega t} F / R T$. The oxidation current density is defined as follows:

$$
j=F D\left(\partial c_{\mathrm{R}} / \partial x\right)_{x=0}
$$

The initial conditions are the same as the boundary conditions in the bulk.

The upper bar of $c_{\mathrm{i}}$ below denotes the Laplace transform of $c_{\mathrm{i}}$. Carrying out the Laplace transformations of eqn (A1)-(A3) and solving the ordinary differential equations satisfied with the boundary conditions, we obtain an expression for the concentrations at the electrode surface:

$$
\begin{aligned}
& \left(\overline{c_{\mathrm{R}}}\right)_{x=0}=c_{\mathrm{R}}^{*} / s-\bar{j} / F \sqrt{s D} \\
& \left(\overline{c_{\mathrm{O}}}\right)_{x=0}=c_{\mathrm{O}}^{*} / s-\bar{j} / F \sqrt{s D}
\end{aligned}
$$

Their inverse Laplace transforms are given by the following expressions:

$$
\begin{aligned}
& \left(c_{\mathrm{R}}\right)_{x=0}=c_{\mathrm{R}}^{*}-\frac{1}{F \sqrt{\pi D}} \int_{0}^{t} \frac{j(u)}{\sqrt{t-u}} \mathrm{~d} u \\
& \left(c_{\mathrm{O}}\right)_{x=0}=c_{\mathrm{O}}^{*}-\frac{1}{F \sqrt{\pi D}} \int_{0}^{t} \frac{j(u)}{\sqrt{t-u}} \mathrm{~d} u
\end{aligned}
$$

Inserting eqn (A5) into (A2) yields,

$$
\frac{1}{c_{\mathrm{O}}^{*} F \sqrt{\pi D}} \int_{0}^{t} \frac{j(u)}{\sqrt{t-u}} \mathrm{~d} u=\frac{\exp \left(\zeta_{\mathrm{ac}}(t)\right)-1}{\exp \left(\zeta_{\mathrm{ac}}(t)+\zeta_{\mathrm{dc}}\right)+1}
$$

where $\zeta_{\mathrm{dc}}$ is the dimensionless dc potential given by $\left(E_{\mathrm{dc}}-E^{\mathrm{o}}\right) F / R T=c_{\mathrm{O}}^{*} / c_{\mathrm{R}}^{*}$. The integral equation with Abel's type can be solved into

$$
\frac{j}{c_{\mathrm{O}}^{*} F \sqrt{D}}=\frac{\mathrm{d}}{\mathrm{d} t} \int_{0}^{t} \frac{\exp \left(\zeta_{\mathrm{ac}}(t-u)\right)-1}{1+\beta \exp \left(\zeta_{\mathrm{ac}}(t-u)\right)} \frac{\mathrm{d} u}{\sqrt{\pi u}}
$$

where $\beta=\exp \left(\zeta_{\mathrm{dc}}\right)$.

Applying Leibniz's theorem for the differentiation of an integral ${ }^{72}$ to eqn (A7), the following equation was obtained:

$\frac{j}{c_{\mathrm{O}}^{*} F \sqrt{D}}=\int_{0}^{t}\left[\frac{\partial}{\partial t} \frac{\exp \left(\zeta_{\mathrm{ac}}(t-u)\right)-1}{1+\beta \exp \left(\zeta_{\mathrm{ac}}(t-u)\right)}\right] \frac{\mathrm{d} u}{\sqrt{\pi u}}+\frac{\exp (0)-1}{1+\beta \exp (0)} \frac{1}{\sqrt{\pi t}}$

Using the following differentiations for $X=\exp \left(\zeta_{\mathrm{ac}}(t-u)\right)$ :

$$
\begin{aligned}
& \frac{\partial X}{\partial t} \equiv \frac{\partial \exp \left(\zeta_{\mathrm{ac}}(t-u)\right)}{\partial t}=\mathrm{i} \omega \zeta_{\mathrm{ac}}(t-u) \exp \left[\zeta_{\mathrm{ac}}(t-u)\right] \\
& \frac{\partial}{\partial X} \frac{X-1}{\beta X+1}=\frac{\beta+1}{\left(\beta \exp \left(\zeta_{\mathrm{ac}}(t-u)\right)+1\right)^{2}}
\end{aligned}
$$

Then, eqn (A8) can be rewritten as follows:

$$
\frac{j}{c_{\mathrm{O}}^{*} F \sqrt{D}}=\mathrm{i} \omega \int_{0}^{t}\left[\frac{\zeta_{\mathrm{ac}}(t-u) \exp \left(\zeta_{\mathrm{ac}}(t-u)\right)(\beta+1)}{\left(\beta \exp \left(\zeta_{\mathrm{ac}}(t-u)\right)+1\right)^{2}}\right] \frac{\mathrm{d} u}{\sqrt{\pi u}}
$$

When we use the conventional approximation $\zeta_{\mathrm{ac}}<1$, we get $\mathrm{e}^{z} \approx 1+z+z^{2} / 2$ for $z(t)=\zeta_{\mathrm{ac}}(t)=\left(F V_{0} / R T\right) \mathrm{e}^{\mathrm{i} \omega t}$ to obtain,

$$
\begin{aligned}
\frac{j}{c_{\mathrm{O}}^{*} F \sqrt{D}}= & \frac{\mathrm{i} \omega}{(\beta+1)} \int_{0}^{t}\left[z(t-u)+\frac{1-\beta}{\beta+1} z^{2}(t-u)\right. \\
& \left.+\left(\frac{1}{2}-\frac{3 \beta}{(1+\beta)^{2}}\right) z^{3}(t-u)+\cdots\right] \frac{\mathrm{d} u}{\sqrt{\pi u}}
\end{aligned}
$$

The integral of $\mathrm{e}^{-a u}(a / \pi u)^{1 / 2}$ from 0 to $t$ becomes the error function, $\operatorname{erf}\left((a t)^{1 / 2}\right)$. Since the asymptotic form of the error function is $\operatorname{erf}\left((\mathrm{i} \omega t)^{1 / 2}\right) \sim 1-\pi^{-1 / 2}(\mathrm{i} \omega t)^{-1 / 2} \exp \left[(\mathrm{i} \omega t)^{1 / 2}\right]$, it tends to unity for a long application of the ac-voltage. Then, eqn (A9) is reduced to

$$
\frac{j}{c_{\mathrm{O}}^{*} F \sqrt{D}}=\frac{\sqrt{\mathrm{i} \omega}}{\beta+1}\left(\zeta_{\mathrm{ac}}+\frac{1-\beta}{\beta+1} \frac{\zeta_{\mathrm{ac}}^{2}}{\sqrt{2}}+\left(\frac{1}{2}-\frac{3 \beta}{(1+\beta)^{2}}\right) \frac{\zeta_{\mathrm{ac}}{ }^{3}}{\sqrt{3}}\right)
$$

When multiplying eqn (A10) by $c_{\mathrm{O}}^{*} / c_{\mathrm{O}}^{*}+c_{\mathrm{R}}^{*}$ and applying the following relationships: $c_{\mathrm{O}}^{*}+c_{\mathrm{R}}^{*}(\beta+1) / c_{\mathrm{O}}^{*}=4 \cosh ^{2}\left(\zeta_{\mathrm{dc}} / 2\right)$, $(1-\beta) /(1+\beta)=-\tanh \left(\zeta_{\mathrm{dc}} / 2\right)$, and $(1+\beta)^{2} / 3 \beta=(4 / 3) \cosh ^{2}\left(\zeta_{\mathrm{dc}} / 2\right)$, we obtain

$$
\frac{j}{\left(c_{\mathrm{O}}^{*}+c_{\mathrm{R}}^{*}\right) F \sqrt{D} \sqrt{\mathrm{i} \omega}}=\frac{\zeta_{\mathrm{ac}} \mathrm{e}^{\mathrm{i} \omega t}}{4} \operatorname{sech}^{2}\left(\zeta_{\mathrm{dc}} / 2\right)\left(G_{1}+G_{2} \zeta_{\mathrm{ac}}+G_{3} \zeta_{\mathrm{ac}}{ }^{2}\right)
$$

where

$$
G_{1}=1, G_{2}=-\frac{1}{\sqrt{2}} \tanh \frac{\zeta_{\mathrm{dc}}}{2}, G_{3}=\frac{1}{2 \sqrt{3}}-\frac{\sqrt{3}}{4 \cosh ^{2}\left(\zeta_{\mathrm{dc}} / 2\right)}
$$

The ac-current density can be expressed as a simple sum of the fundamental frequency and its harmonics. Since $\left|G_{2}\right|$ and $\left|G_{3}\right|$ are independent of $\omega,|j|$ is proportional to $\omega^{1 / 2}$ even if the current includes harmonics. Fig. 10 shows variations of the maximum of the dimensionless admittance densities

$$
Y_{n}=\frac{1}{4} \operatorname{sech}^{2}\left(\zeta_{\mathrm{dc}} / 2\right) \sum_{k=1}^{n} G_{k} \zeta_{0}^{k-1}
$$

with the dc-potential for the ac-amplitude of $10 \mathrm{mM}$. The variation of $Y_{3}$ is close to $Y_{1}$ for the observed current. Harmonics,

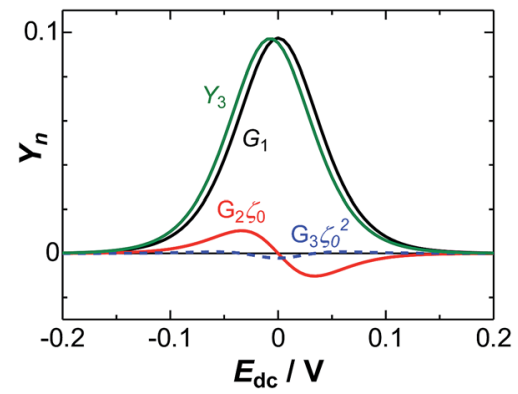

Fig. 10 The variations of $Y_{n}$ with $E_{\mathrm{dc}}$, where $G_{n} \zeta_{0}{ }^{n-1}$ is each component of $Y_{n}$, calculated using eqn (A12) and (A13) for $V_{0}=10 \mathrm{mM}$. 
especially the second harmonics $\left(G_{2} \zeta_{0}\right)$, cause a potential shift of up to $8 \mathrm{mV}$. They have no effect on the peak current.

\section{References}

1 A. N. Frumkin, Z. Phys. Chem., Abt. A, 1933, 164, 121.

2 J. Kuta, Comprehensive Treatise of Electrochemistry: Volume 8 Experimental Methods in Electrochemistry, ed. R. E. White, J. O'. M. Bockris, B. E. Conway and E. Yeager, Plenum Press, New York, 1984, ch. 4, pp. 277-282.

3 A. J. Bard and L. R. Faulkner, Electrochemical Methods, Fundamentals and Applications, John Wiley, New York, 2nd edn, 2001, pp. 571-575.

4 H. H. Bauer, J. Electroanal. Chem., 1966, 12, 64.

5 J. D. McLean and A. Timnick, Anal. Chem., 1967, 39, 1669.

6 A. Aramata and P. Delahay, J. Phys. Chem., 1964, 68, 880.

7 H. Kojima and A. J. Bard, J. Am. Chem. Soc., 1975, 97, 6317.

8 D. Armand and J. Clavilier, J. Electroanal. Chem., 1989, 270, 331.

9 G. Valette, J. Electroanal. Chem., 1988, 255, 225.

10 M. Bacchetta, S. Trasatti, L. Doubova and A. Hamelin, J. Electroanal. Chem., 1988, 255, 237.

11 B. Damaskin, U. Palm and M. Salve, J. Electroanal. Chem., 1987, 218, 65.

12 A. M. Gómez-Marín and J. M. Feliu, Electrochim. Acta, 2012, $82,558$.

13 S. Levine, K. Robinson and W. R. Fawcett, J. Electroanal. Chem., 1974, 54, 237.

14 M. A. Vorotyntsev and P. V. Mityushev, Electrochim. Acta, 1991, 36, 401.

15 H. Wang and L. Pilon, Electrochim. Acta, 2012, 63, 55.

16 J. Lim, J. Whitcomb, J. Boyda and J. Varghese, J. Colloid Interface Sci., 2007, 305, 159.

17 M. S. Kilic, M. Z. Bazant and A. Ajdari, Phys. Rev. E: Stat., Nonlinear, Soft Matter Phys., 2007, 75(021503), 1.

18 R. P. Buck, J. Electroanal. Chem., 1973, 46, 1.

19 M. C. Henstridge, E. J. F. Dickinson and R. G. Compton, Chem. Phys. Lett., 2010, 485, 167.

20 M. Huske, R. Stockmann, A. Offenhausser and B. Wolfrum, Nanoscale, 2014, 6, 589.

21 D. J. Gavaghan and S. W. Feldberg, J. Electroanal. Chem., 2000, 491, 103.

22 L. I. Daikhin, A. A. Kornyshev and M. Urbakh, Electrochim. Acta, 1997, 42, 2853.

23 E. J. F. Dickinson and R. G. Compton, J. Electroanal. Chem., 2011, 661, 198.

24 W. J. Royea, O. Krüger and N. S. Lewis, J. Electroanal. Chem., 1997, 438, 191.

25 W. R. Fawcett, G. J. Chavis and M. Hromadová, Electrochim. Acta, 2008, 53, 6787.

26 P. Kulesza, T. Jędral and Z. Galus, J. Electroanal. Chem., 1980, 109, 141.

27 J. Nerut, P. Möller and E. Lust, Electrochim. Acta, 2004, 49, 1597.

28 R. Jäger, E. Härk, P. Möller, J. Nerut, K. Lust and E. Lust, J. Electroanal. Chem., 2004, 566, 217.
29 S. W. Barr, K. L. Guyer and M. J. Weaver, J. Electroanal. Chem., 1980, 111, 41.

30 W. R. Fawcett, M. Hromadova, G. A. Tsirlina and R. R. Nazmutdinov, J. Electroanal. Chem., 2001, 498, 93.

31 K. Nisancioglua and J. Newman, J. Electrochem. Soc., 2012, 159, E59.

32 S.-L. Wua, M. E. Orazem, B. Tribollet and V. Vivier, Electrochim. Acta, 2014, 131, 3.

33 M. van Soestbergen, Russ. J. Electrochem., 2012, 48, 570.

34 S. Levine, J. Colloid Interface Sci., 1971, 37, 619.

35 R. Guidelli and M. L. Foresti, Electrochim. Acta, 1973, 18, 301.

36 W. R. Fawcett and S. Levine, J. Electroanal. Chem., 1973, 43, 175.

37 R. Guidelli, J. Electroanal. Chem., 1976, 74, 347.

38 K. Aoki, Y. Hou, J. Chen and T. Nishiumi, J. Electroanal. Chem., 2013, 689, 124.

39 X. Zhao, K. J. Aoki, J. Chen and T. Nishiumi, RSC Adv., 2014, 4, 63171.

40 Y. Hou, K. J. Aoki, J. Chen and T. Nishiumi, Univers. J. Chem., 2013, 1, 162.

41 H. Wang, K. J. Aoki, J. Chen, T. Nishiumi, X. Zeng and X. Ma, J. Electroanal. Chem., 2015, 741, 114.

42 Y. Hou, K. J. Aoki, J. Chen and T. Nishiumi, J. Phys. Chem. C, 2014, 118, 10153.

43 K. J. Aoki, Electrochim. Acta, 2016, 188, 545.

44 K. J. Aoki, J. Electroanal. Chem., 2016, 779, 117.

45 T. Pajkossy, T. Wandlowski and D. M. Kolb, J. Electroanal. Chem., 1996, 414, 209.

46 T. Pajkossy, Solid State Ionics, 1997, 94, 123.

47 Z. Kerner and T. Pajkossy, Electrochim. Acta, 2002, 47, 2055. 48 T. Pajkossy and D. M. Kolb, Electrochim. Acta, 2009, 54, 3594. 49 T. Pajkossy and D. M. Kolb, Russ. J. Electrochem., 2009, 45, 29. 50 M. H. Martin and A. Lasia, Electrochim. Acta, 2011, 56, 8058. 51 W. Schelder, J. Phys. Chem., 1975, 79, 127.

52 J. Newman, J. Electrochem. Soc., 1970, 117, 198.

53 J. B. Jorcin, M. E. Orazem, N. Pebere and B. Tribollet, Electrochim. Acta, 2006, 51, 473.

54 V. M. W. Huang, V. Vivier, M. E. Orazem, N. Pebere and B. Tribollet, J. Electrochem. Soc., 2007, 154, C99.

$55 \mathrm{~S}$. L. Wu, M. E. Orazem, B. Tribollet and V. Vivier, J. Electrochem. Soc., 2009, 156, C214.

56 A. Le Méhauté and G. Crépy, Solid State Ionics, 1983, 9/10, 17. 57 T. Pajkossy, J. Electroanal. Chem., 1991, 300, 1.

58 M. Keddam and H. Takenouti, Electrochim. Acta, 1988, 33, 445.

59 T. Pajkossy and L. Nyikos, J. Electrochem. Soc., 1986, 133, 2061. 60 E. Warburg, Annu. Rev. Phys. Chem., 1899, 67, 493.

61 Impedance Spectroscopy, ed. J. R. Macdonald, Wiley, New York, 1987.

62 J.-P. Diard, B. L. Gorrec and C. Montella, Handbook of Electrochemical Impedance Spectroscopy, 2012, http://www. bio-logic.info/assets/handbook\%20of\%20EIS/20130801\% 20-\%20Zdiffusion.pdf.

63 A. Lasia, in Modern Aspects of Electrochemistry, ed. R. E. White, B. E.Conway and J. O'. M. Bockris, Kluwer Academic/Plenum Publishers, New York, 1999, vol. 32, p. 143. 
64 L. Nyikos and T. Pajkossy, Electrochim. Acta, 1985, 30, 1533. 65 G. J. Brug, A. L. G. Van Den Eeden, M. Sluyters-Rehbach and J. H. Sluyters, J. Electroanal. Chem., 1984, 176, 275.

66 P. Zoltowski, J. Electroanal. Chem., 1998, 443, 149.

67 A. M. Bratkovsky and A. P. Levanyuk, Phys. Rev. B: Condens. Matter Mater. Phys., 2001, 63, 132103.

68 P. Zubko, J. C. Wojdeł, M. Hadjimichael, S. Fernandez-Pena, A. Sené, I. Luk'yanchuk, J.-M. Triscone and J. Íñiguez, Nature, 2016, 534, 524.
69 C. M. Krowne, S. W. Kirchoefer, W. Chang, J. M. Pond and L. M. B. Alldredge, Nano Lett., 2011, 11, 988.

70 D. J. Griffiths, Introduction to Electrodynamics, Prentice-Hall, Inc., 2nd edn, 1989, pp. 121-123.

71 A. S. Cuharuc, G. Zhang and P. R. Unwin, Phys. Chem. Chem. Phys., 2016, 18, 4966.

72 M. Abramowitz and I. A. Stegun, Handbook of Mathematical Functions, National Bureau of Standards, 1972, p. 11, eqn (3.3.7). 